\title{
Les TICE, un moyen d'émancipation didactique au Maroc
}

Asmaâ AFNAKAR, Université Chouaib Doukkali, El Jadida, Maroc

\section{Introduction}

Cette communication se propose de faire connaitre les résultats d'une expérience d'écriture collaborative qui a été menée dans le cadre de la formation des enseignants du français dans le Centre Régional des Métiers de l'Éducation et de la Formation (CRMEF) de Kénitra au Maroc et que nous inscrivons sous le signe de la transgression afin de la relier au thème du colloque.

Ainsi, sur la base de cette expérience d'écriture, d'enrichissement, d'échange et de partage démarrée sur un blog, mais, pour des raisons techniques, menée sur un réseau social, notre contribution tentera de démontrer que les TICE pourraient être un moyen transgressif d'esquiver les contraintes institutionnelles et les contraintes imposées par la nature de l'enseignement-apprentissage d'une langue étrangère pour créer une zone connexe à la classe où se prolongeraient les activités entreprises pendant la séance de cours.

Avant d'exposer notre expérience et pour mieux en faire ressortir le caractère transgressif, nous commencerons par présenter un bref aperçu du contexte éducatif marocain et notamment de l'enseignement du français au Maroc et la place qui y est accordée aux TICE. Nous exposerons par la suite notre expérience, mes professeurs-stagiaires et moi, en vue de montrer que ces technologies permettent une transgression et une redéfinition du temps didactique, du milieu d'enseignement-apprentissage et des rapports entre enseignant et apprenant.

\section{Cadre}

\section{1-Aperçu du système éducatif marocain}

Le système éducatif marocain est en perpétuelle mutation. Depuis l'indépendance en 1956, les réformes se sont succédé dans une tentative de mettre en place des contenus, des démarches et des méthodes qui répondent parfois à des choix politiques, comme c'était le cas pour l'arabisation et la marocanisation du corps enseignant après l'indépendance ; ou alors qui satisfont aux besoins de la société (la scolarisation des filles, l'éducation non-formelle...) et aux exigences de la sphère scientifique et concourent avec l'évolution continue des tendances didactico-pédagogiques dans le monde (intégration des méthodes actives, par exemple). Dans ce sens, les textes officiels qui régissent l'enseignement dans les cycles 
secondaires (le collège et le lycée) sont passés du statut d'instructions à celui de recommandations en 1991, pour porter l'étiquette d'orientations en 2009.

Force est de remarquer que le mot «instructions » revêt un caractère prescriptif, celui de « recommandations » tend vers l'incitatif. Les « orientations », quant à elles, ont plutôt une facture indicative. Ce changement laisse entendre que les enseignants ont acquis petit à petit un grand «pouvoir transgressif » : une grande possibilité de l'initiative. De même pour les dispositifs de formation des enseignants mis en place ces dernières années, qui tout en prônant une méthodologie, insistent sur la marge de liberté laissée au formateur et au futur enseignant pour forger ses propres procédés d'enseignement-apprentissage, en témoigne notre projet d'écriture collaborative qui ne figure pas dans le dispositif de formation des enseignants du collège. Toutefois, d'autres contraintes semblent réduire, voire anéantir cette marge de liberté, notamment dans l'enseignement du français.

En effet, la nature du français à enseigner qui vacille entre FLE et FLS rend les enseignants perplexes quant aux méthodes à adopter. Au Maroc, le statut du français n'est pas bien déterminé. Fouzia Benzakour précise que «la perception ambiguë du français (l'élite francisée y est attachée par des réseaux de toute nature, alors que les partisans de l'arabisation le contestent en tant qu'idiome véhiculaire) lui vient à la fois de son passé colonial et de son statut jamais clairement défini, preuve qu'une politique d'aménagement linguistique transparente a du mal à prendre pied au Maroc. » (51) Elle ajoute que «même la Charte nationale d'éducation et de formation, promulguée en 1999, et qui consacre deux articles à la maîtrise des langues étrangères, ne dit pas explicitement mais sous-entend seulement que le français est la première langue étrangère (LE). Il s'agit donc bien d'un statut de fait et non de jure. » (51) Effectivement, dans la Charte nationale de l'éducation et de la formation, il est clairement dit, à propos des langues enseignées, que hormis l'arabe et l'amazigh, toutes les autres langues sont considérées comme des LE : le français, l'anglais, l'italien, l'espagnol, l'allemand ... Néanmoins, il est à mentionner à ce niveau que seul le français est enseigné dès le primaire, voire dès le préscolaire dans le secteur privé ; l'anglais, quant à lui, est enseigné à partir du collège et les autres langues sont dispensées à partir du lycée. S'il est dit, dans les textes officiels, que le français est une LE, il suffit de rappeler qu'il est la langue d'enseignement dans le cycle supérieur (dans les universités et les instituts supérieurs) pour comprendre que cette langue reste largement favorisée par rapport aux autres langues étrangères enseignées dans les écoles marocaines. Mieux encore, le français enseigné dans le cycle secondaire qualifiant (le lycée) et les méthodes qui y sont adoptées n'ont rien à envier à celles utilisées en France. Les supports sont des œuvres intégrales d'une qualité littéraire et 
intellectuelle incontestable : Candide de Voltaire, Le père Goriot de Balzac, des nouvelles de Maupassant, Le bourgeois gentilhomme de Molière... Pour étudier ces œuvres, les enseignants ont recours à la lecture méthodique, l'explication linéaire, la lecture sélective, la lecture analytique, le commentaire composé... En d'autres mots, le statut du français dans ce cycle frôle le FLM.

A cette ambiguïté, s'ajoute le carcan du temps lié aux programmes : ne dépassant pas les quatre heures par semaine au collège, par exemple, le temps imparti aux activités de réception et de conceptualisation de production orale et écrite laisse peu de place à l'initiative émancipatrice. Dès lors, la liberté didactique offerte aux enseignants devient plus responsabilisante que gratifiante et le caractère des textes officiels pourrait passer de celui de contraignant à celui de salutaire.

Ce qui précède nous mène à dire qu'au Maroc, il serait difficile de parler de transgression puisque les textes officiels, mis à part les finalités éducatives et les compétences à installer pour chaque cycle, n'imposent aucune norme didactique. En quoi notre expérience est-elle donc transgressive ?

\section{2- Le recours aux TICE au Maroc, une transgression didactique?}

Faire appel aux nouvelles technologies d'information et de communication en classe marocaine relèverait-il de la transgression didactique ? La réponse est double : non et oui.

Le recours aux TICE pourrait ne pas être considéré comme transgressif, du moment que le Maroc a gagé sur ces technologies comme l'un des catalyseurs de la réforme. La charte nationale d'éducation et de formation insiste sur l'initiation des apprenants aux TICE, dès le second cycle de l'école primaire (article 66) : elle leur consacre le dixième levier. Néanmoins, l'usage qui en est recommandé est très réducteur. Il y est stipulé que le recours à ces outils se fera principalement en matière de formation continue et comme une solution pour remédier aux problèmes liés à l'éloignement et à l'enclavement des apprenants et des enseignants, puisqu'elles offrent la possibilité de l'enseignement à distance.

Les TICE ne sont donc pas considérées comme un moyen d'optimiser la formation en présentiel. En outre, elles sont intégrées dans l'enseignement avec une grande circonspection, car elles ne sauraient remplacer l'enseignant ni déroger aux rapports traditionnels entre professeur et élève définis comme «la véritable relation pédagogique fondatrice de l'acte éducatif: la relation vivante maître-élève, basée sur la compréhension et le respect » (C.O.S.E.F. article 119).

De même, les Orientations Pédagogiques des enseignants du collège ${ }^{1}$ encouragent les professeurs à utiliser ces technologies. Ainsi, il est bien souligné que «chaque fois que les 
conditions matérielles le permettent, on n'hésitera pas à avoir recours aux nouvelles technologies pour aider les élèves à apprendre et développer une autonomie utile, qui favorise une utilisation judicieuse de ces nouveaux moyens» (M.E.N. 14). De plus, elles leur consacrent toutes une entrée sous le titre «L'intégration des TIC à l'enseignement du français au collège » (M.E.N., O.P., 38) où sont dénombrés leurs avantages. Plusieurs tâches nécessitant le recours aux TICE y sont également proposées avec les différents usages qui pourraient en être faits en classe de langue. Parallèlement, sont signalées aussi les difficultés pouvant survenir lors de leur usage. À la tête de ces difficultés se trouve l'éventuelle surcharge du travail et les problèmes relatifs à la gestion de la classe que peuvent occasionner ces outils. Il est certain que s'écarter des modes de fonctionnement traditionnels et installer de nouveaux environnements d'apprentissage constituent en soi une immense tâche. S'ajoutent à tout cela les difficultés susceptibles d'être posées par les problèmes de gestion de la classe où sont intégrées ces technologies. Comme le souligne Pascal Bihouée, «L'utilisation des nouvelles technologies favorise le travail interactif, le travail sur projet, le travail en équipe ; mais elle modifie aussi [la] relation [de l'enseignant] aux élèves et à la classe » (153). L'enseignant risque de perdre de son contrôle de la classe et doit gérer plusieurs éléments en même temps : venir en aide aux élèves qui se sentiraient en désarroi face aux outils utilisés, surveiller l'accès aux sites Internet, faire attention aux apprenants «zappeurs », gérer le temps...

Tous les textes officiels sont donc unanimes pour dire que les TIC sont à intégrer dans l'enseignement, mais prudemment ; elles doivent participer à une évolution mais non à une révolution. L'expérience que nous allons exposer constitue une transgression de cette conception de l'usage modéré à faire des TIC préconisé dans ces textes.

\section{Protocole méthodologique}

\section{1- présentation du dispositif didactique}

Le projet d'écriture collaborative a été mené avec un groupe de vingt-huit professeursstagiaires qui suivaient leur formation dans le C.R.M.E.F de Kénitra (Maroc).

Destinés à enseigner dans le cycle secondaire collégial, les stagiaires étaient amenés à animer des cours de production écrite et à gérer des projets d'écriture. L'un des objectifs de ce projet était de les faire passer par cette épreuve pour qu'ils puissent en mesurer aussi bien l'intérêt que la difficulté. Ayant un module consacré à l'usage des TICE, nous avons décidé d'intégrer cet élément à notre projet d'écriture et de mener une sorte d'expérience 
pronostique pour étudier la faisabilité technique d'un projet d'écriture collaborative avec des élèves du collège.

Après l'étude de plusieurs propositions des stagiaires, le groupe a opté pour le pastiche d'un roman de littérature de jeunesse que les professeurs-stagiaires ont découvert dans le module dédié à la lecture. Les raisons qui sont à la source de ce choix sont les suivantes :

Le roman en question, L'enfant océan de Mourlevat, s'inscrit dans la mouvance controversée de la littérature de jeunesse. Il a eu beaucoup de succès. Il constitue une réécriture-réappropriation du conte du Petit Poucet. Une expérience similaire de détournement de contes pourrait s'avérer amusante et intéressante pour les stagiaires et leurs lecteurs. Elle développerait chez eux des appétences de lecture : en général, ce genre d'écrit pique la curiosité des lecteurs et les pousse à remonter à l'œuvre réécrite ainsi qu'aux autres versions disponibles du conte. Il permet aussi de découvrir d'autres contes qui peuvent croiser leurs lectures.

Ce roman est sous-tendu par un relais narratif dont le pastiche permettrait de passer par une étape de travail coopératif (avec partage des tâches) et ainsi faire participer tous les membres du groupe de manière individuelle ou en binôme, au départ, avant d'arriver à l'écriture collaborative. Il est à rappeler à ce niveau que l'« on parle de travail collaboratif lorsque plusieurs personnes travaillent ensemble sur le même sujet: chacun met ses compétences à profit pour améliorer le projet et optimiser sa réussite (Wikipédia est un exemple de plate-forme collaborative). Quant au travail coopératif, il correspond à une tâche réalisée par plusieurs personnes qui se sont réparti au préalable les rôles. Dans ce cas, il n’y a pas de mutualisation » (Bihouée et Colliaux.134)

Le relais narratif, dans ce roman, permet de développer les stratégies métacognitives $^{2}$ chez le jeune lecteur, d'où son intérêt. L'histoire est présentée comme un puzzle dont il faut recoller les morceaux pour appréhender la trame.

Le roman est écrit dans un style simple et très intelligible pour des collégiens. Notre objectif va dans la même perspective : réaliser une production écrite accessible au collégien marocain dont le sens peut être aisément saisi.

\section{2- Les étapes retenues pour ce projet :}

Les heures allouées aux ateliers ne dépassent pas le nombre de deux par semaine et sont respectivement réservées à trois activités: l'écriture, la lecture et la communication orale. Les formateurs responsables de ces ateliers gèrent entre eux la répartition de ces séances. Par conséquent, après la présentation du projet d'écriture longue à mener en groupe, le volume 
horaire imparti à l'atelier d'écriture n'étant pas suffisant pour planifier, gérer et évaluer ce projet, nous nous sommes mis d'accord pour y intégrer les TICE, notamment Internet, versant ainsi dans l'écriture collaborative. Nous avons donc décidé le planning suivant :

Pour le premier semestre :

1- $\quad$ Lecture et étude du roman à pasticher ;

2- $\quad$ Ouverture d'un blog où s'opérera l'échange entre les stagiaires et attribution de pseudonymes $^{3}$ (mais, pour des raisons techniques, et ce sont là les aléas de ces technologies, nous avons dû basculer sur un réseau social où la majorité des stagiaires était déjà inscrite);

3- Choix du conte à détourner et proposition de trames susceptibles d'être développées ;

4- Choix collectif d'une histoire ;

5- Partage des tâches ;

Pour le second semestre :

6- $\quad$ Partage, correction et validation des productions écrites sur le blog ;

7- $\quad$ Publication du produit;

8- Évaluation du projet.

Les séances en présentiel servaient surtout à recadrer le travail et à évaluer chaque étape.

\section{Aspects de la transgression}

\section{1- Transgression des textes officiels}

Cette expérience ne respecte ni l'usage des TICE stipulé dans la charte nationale ni celui proposé dans les orientations pédagogiques et ne correspond non plus à la conception de l'activité de production écrite qui y présentée.

En effet, nous avons eu recours à Internet et notamment à un réseau social pour développer et affiner notre production en dehors de la salle de cours. Ceci nous a permis d'abolir les limites de la classe et de créer un espace plus grand s'étendant au-delà des murs de notre centre de formation et s'actualisant à chaque fois que l'enseignant ou l'apprenant se connectent pour consulter la page du groupe ou pour partager un commentaire, une remarque ou une production.

S'agissant de l'activité d'écrire, elle se présente dans les orientations pédagogiques du secondaire collégial sous une forme mitigée : elle s'inspire des méthodes traditionnelles et tend cependant vers le renouveau. Il est bien noté dans ce texte à l'adresse des enseignants que la séance dédiée à l'expression écrite «devrait normalement constituer l'aboutissement de tout le travail réalisé en amont et le creuset où viennent se mêler toutes les formes d'acquis antérieurs (de lecture, de grammaire, de communication...) » (M.E.N. 29). Nous sommes de 
plain-pied dans la conception traditionnelle de l'écrit qui crée chez l'apprenant une représentation rebutante de l'activité d'écrire. Comme le souligne Yves Reuter, "l'écriture n'est pas enseignée en tant que telle. Elle se présente de fait comme une synthèse 'magique' des autres enseignements, essentiellement les 'sous-systèmes' de la langue: orthographe, syntaxe, vocabulaire, conjugaison... c'est aux élèves à apprendre, par eux-mêmes, comment les intégrer »(15). La production écrite est programmée en fin de semaine et constitue un moment d'intégration des ressources fraîchement installées. Elle est vécue par les élèves et conçue par les enseignants (comme dicté par l'institution) comme un moment d'évaluation. Il s'agit plus d'une mise à l'épreuve pour évaluer le degré d'acquisition des apprentissages que d'un moment d'expression écrite. Contrairement à l'objectif qui lui est assigné : « développer le plaisir d'écrire » (M.E.N. 30), cette activité, elle, est souvent dénuée de plaisir.

La conception de l'activité est très problématique en elle-même : «la situation de communication de la rédaction est tout aussi artificielle et complexe. Il s'agit de s'adresser au professeur, en feignant de ne pas lui écrire et/ou d'écrire à quelqu'un d'autre (explicitement désigné ou non), pour l'informer ou le distraire, tout en sachant que c'est bien à l'enseignant qu'on écrit dans le but principal d'être évalué» (Reuter 16). Quoique l'un des objectifs de cette activité est d'«acquérir la capacité de s'exprimer et de communiquer par écrit» (M.E.N. 30), en réalité, il ne s'agit pas de s'exprimer par écrit mais de faire semblant d'écrire dans une situation de communication simulée et/ou imposée par la consigne. La créativité et l'imaginaire sont donc mis en veilleuse. Par conséquent, étant peu significative pour les élèves, cette activité est vécue tant bien que mal par eux et elle finit par les désintéresser.

Par contre, «l'écriture longue en projet -au travers des expériences connuesmanifeste un certain nombre d'effets positifs: motivation des élèves, développement des compétences relationnelles et organisationnelles, développement des compétences scripturales et textuelles: déblocage vis-à-vis de l'écrit, longueur des textes produits, meilleure structuration globale, prise en compte du destinataire, intérêt des écrits, etc. » (Reuter 27). Nos stagiaires s'exprimaient justement par écrit dans le cadre d'un projet. Lors du moment d'évaluation de notre expérience, ils ont affirmé qu'ils n'avaient pas ressenti le poids de la tâche qu'il leur incombait de réaliser. Plusieurs d'entre eux ont avoué trouver du plaisir à laisser des commentaires et à participer à l'évolution de l'écriture du roman. Deux stagiaires ont même tenté de transposer l'expérience auprès d'un public d'une dizaine d'apprenants collégiens de la première année qui appartenaient à un établissement d'accueil où ces stagiaires effectuaient leur mise en situation professionnelle. Ils ont encadré pendant un mois l'écriture collaborative d'un conte d'une quarantaine de lignes. Sachant que ces 
apprenants produisaient, tant bien que mal, en classe et en petits groupes, six lignes au grand maximum. Les élèves collégiens ont laissé une soixante de lignes de commentaires, de demandes d'explication ou d'aide. De ce fait, une remise en question des soubassements didactiques de la séance de production écrite s'impose.

\section{2- Transgression du temps didactique}

Il est difficile de compter, de manière précise, les heures que les professeurs-stagiaires ont passées devant leurs ordinateurs pour écrire et lire les différentes productions et pour consulter la page du groupe. Ils ne se connectaient pas tous à la même fréquence. Le nombre d'heures variait selon la motivation des participants. Ce qui est sûr cependant, c'est que tous les intervenants ont passé, en moyenne, plus que le triple des heures allouées à l'atelier d'écriture. Cette expérience a donc permis la création d'un espace plus convivial que la salle de cours qui, se basant sur des outils de communication asynchrone, permettait à chacun d'intervenir à son rythme et à sa guise. Considérons l'échange suivant:

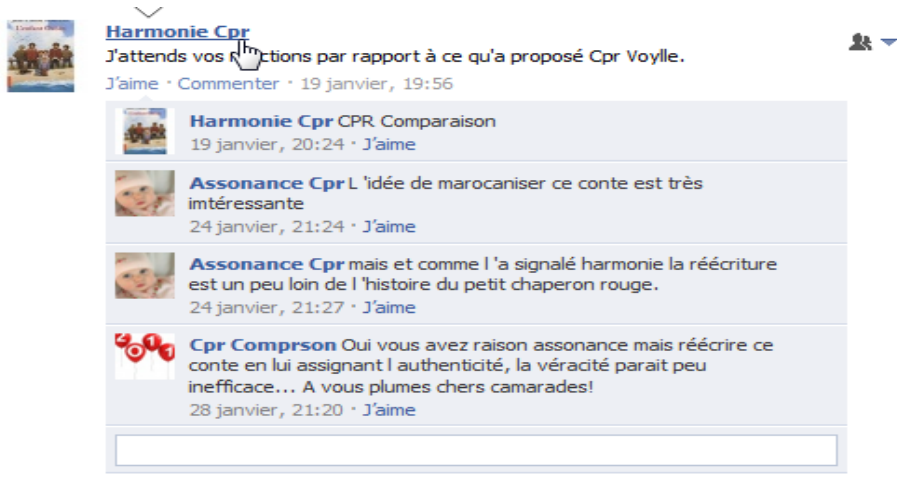

Figure 1 : échange des participants sur la page du groupe sur un réseau social (prise d'écran)

Tous les messages ont été postés le soir, mais avec des intervalles de deux à quatre jours.

À l'instar des stagiaires, nous avons dû nous connecter et consulter notre page plusieurs fois par jour. Ce genre de projet exige une grande disponibilité de la part de l'enseignant. Il nécessite de lui de longues heures de connexion à Internet qui dépassent de loin les heures mentionnées sur son tableau de service. La séance de cours se prolonge aux dépens de son temps libre, puisqu'il doit répondre aux commentaires, gérer les conflits, motiver les apprenants...

\section{3- transgression de la conception traditionnelle du triangle didactique}

Le projet d'écriture collaborative exige une redéfinition des rôles de l'enseignant et de son public. L'enseignant doit ainsi avoir comme souci permanent la motivation de ses apprenants. Il doit les faire travailler sans les brusquer. Ses compétences de pédagogue sont 
tout le temps mises à l'épreuve. S'il gère le projet, il n'en est pas le maître : il doit se contenter du rôle de «manager » et savoir remédier aux imprévus. Ceci passe nécessairement par une remise en question et une transgression de la conception traditionnelle du triangle didactique. L'enseignant perd de son autorité. Mieux encore, le recours aux réseaux sociaux, comme c'était le cas pour nous, favorise l'«humanisation» des participants et plus particulièrement du professeur, car il permet l'accès à plusieurs informations personnelles, ne serait-ce qu'aux centres d'intérêt de l'individu. L'image de l'enseignant perd de son opacité et gagne en affectivité.

En somme, notre premier défi était de créer une atmosphère d'entente et de fortifier les rapports entre les différents membres de la classe, car les tensions déteignent sur le travail en groupe.

\section{De la transgression à la progression}

Ce projet d'écriture longue, quoique ne se pliant pas à l'usage des TIC préconisé dans les textes institutionnels, nous a permis de réaliser quelques résultats auxquels nous ne pouvions aspirer en classe. Donnons à titre d'exemple la participation et l'interaction de la majorité du groupe. En témoigne la figure suivante :

\section{Didactique du français}

\section{notre projet démarre!}

Par harmonie

Voici comme promis un pett espace oủ nous pourrons échanger autour de notve projet d'écrive collaborative. Un pett rappel : notve projet se déroulera en deux temps :

1. Dans un premier temps, on se penchera de plus press sur le roman de Jean-Claude Mourlevat, en foccumence l'Enfont Océon. On en fera une étude narratologique, qui nous éclairen sur les choix à opérer pour Yécriture de notre roman.

2. Dans un devième temps, et après s'être mis d'accord sur la tame, les personnages et les autres éléments du réct, on passera à lécriture.

Pour commencer, $\vec{l}$ a imera is bien recuelili vos impressions de lecture.

Nhéstez pas a laisser des commentaires, faire des propositions, poser des questions et réagir aux commentaires, propositions et questions de vor camarades!

Commentaires : 62 : : Lien direct

Figure 2 : Message de démarrage du projet sur le blog (prise d'écran) 
Le message de démarrage, à lui seul, a recueilli soixante-deux commentaires. Il nous aurait été difficile de réaliser le même nombre de réactions en classe et, si c'était possible, donner la parole soixante-deux fois, en prenant en considération que certains stagiaires sont plus prolixes que d'autres, nous aurait pris beaucoup de temps. Écrire devant son écran d'ordinateur encourage aussi bien les plus timides que les faibles à intervenir, puisqu'ils ont le temps d'écrire et de soigner leurs messages. Chacun réagit au(x) commentaire(s) qui l'interpelle(nt) le plus au moment où il veut. Certains stagiaires publiaient leur participation au-delà de minuit.

Par ailleurs, d'après Sylvie Plane, il existe cinq grandes catégories de contraintes d'écriture :

- des contraintes d'ordre linguistique : ce sont les règles qui régissent les fonctionnements syntaxiques, sémantiques, morphologiques, prosodiques, etc. ;

- des contraintes d'ordre psycholinguistique imposées par les limites des ressources cognitives du scripteur, son rapport à la langue utilisée pour écrire ;

- des contraintes résultant de prescriptions imposées par la consigne ou que le scripteur s'impose: elles relèvent de différents ordres (linguistiques, thématiques, stylistiques...) et peuvent porter sur toutes les dimensions de la production (aspect matériel, quantité à produire, visée à atteindre...) ;

- des contraintes imposées par le medium de production : l'espace ouvert par la page ou par l'écran d'ordinateur configure pour une certaine part la production du texte à venir. Ainsi, les media de production exercent une influence sur l'activité du scripteur ;

- et des contraintes imposées par le texte produit: au fur et à mesure que le texte est produit, il restreint les choix possibles pour rédiger la suite (57-77).

En optant pour l'écriture collaborative, plusieurs de ces contraintes s'amenuisent. Les contraintes psycholinguistiques s'amoindrissent parce que l'apprenant ne se sent plus évalué et sait qu'il peut perfectionner son écrit chez lui sans contrainte pressante de temps, se faire aider, se concerter avec les autres participants... Les contraintes qui résultent du choix du médium s'affaiblissent aussi, car le scripteur peut écrire sur une page blanche et recopier par la suite sa production sur son ordinateur ou même utiliser les deux en même temps : noter les premières idées sur une page blanche et les développer sur l'outil informatique ou l'inverse.

De surcroit, cette expérience était réellement riche d'apports et a contribué au développement des compétences scripturales des participants et à leur socialisation, car comme le soutient Marlène Lebrun, ce type d'activité « favorise une posture d'auteur et une posture critique, [et] développe de nombreuses compétences tant en lecture qu'en écriture, 
notamment la planification, l'attention à la cohérence et au destinataire à travers la relecture et la réécriture qui sont favorisées par le dispositif » (116). Il permet le décloisonnement des apprentissages et peut inverser le cheminement traditionnel qui part de la lecture pour arriver à l'écrit. L'écriture collaborative nourrit des appétences de lecture chez les apprenants qui cherchent à perfectionner leurs productions en vue de satisfaire leurs lecteurs, et partant font des recherches et des lectures individuelles supplémentaires, sans parler des lectures qu'ils font des écrits de leurs camarades non seulement pour les découvrir, mais également pour en donner leur avis, les corriger, les réécrire ou les enrichir.

De fait, plusieurs professeurs-stagiaires ont confirmé avoir fourni des efforts pour être à la hauteur du projet. Ils ont été amenés à revoir leurs leçons de grammaire et de conjugaison à plusieurs reprises pour corriger et perfectionner leurs écrits. Cette étude contextualisée conférait un sens aux leçons révisées, d'où l'intérêt de mener ce genre d'activité sous la bannière de la pédagogie du projet. Si ce genre d'écriture longue avait été mené avec une classe du collège, il aurait permis de programmer des leçons de langue dont le besoin aurait été perçu par les apprenants et dont l'acquisition aurait été mise au service du projet. Ainsi, les apprentissages deviennent fonctionnels et acquièrent un sens aux yeux des élèves.

Les stagiaires soutenaient également que ce genre de production leur permettait de se concerter, d'échanger et de travailler en groupe. La socialisation des apprenants est donc l'un des avantages de l'apprentissage par le biais de projets.

\section{Conclusion}

Les TICE, mises au service de l'écriture collaborative ou d'autres projets, sont un moyen didactique efficient pour l'enseignement-apprentissage du français au Maroc, du moment qu'elles favorisent la transgression des limites spatio-temporelles et de certaines restrictions prescrites dans les textes officiels.

Les contraintes temporelles liées au volume horaire alloué à l'enseignement du français s'annihilent, notamment pour l'activité d'écriture qui est problématique de par sa nature. Le choix d'Internet comme médium d'écriture et de partage permet à tout un chacun de produire à son rythme.

Écrire en projet de collaboration aide les apprenants à ne plus se représenter la séance de production comme un moment éprouvant d'évaluation : l'intégration des TIC agrémente l'acte scriptural. Néanmoins, il est à souligner qu'elles exigent une bonne scénarisation pédagogique pour la réussite de leur intégration dans les séances de cours. 


\section{Bibliographie}

Bihouée, Pascal. Cours et supports : l'art de préparer sa classe. Paris : Organisations, 2009.

--- et Anne Colliaux. Enseigner différemment avec les TICE. Groupe Eyrolles, 2011.

Benzakour, Fouzia. «Langue française et langues locales en terre marocaine : rapports de force et reconstructions identitaires ». Hérodote 126 (2007) : 45-56.

Commission spéciale d'éducation et de formation (COSEF). Charte nationale d'éducation et de formation. Maroc, 1999.

Lebrun, Marlène, dir. La classe de français et de littérature. Bruxelles : E.M.E., 2010.

Leroux, Serge, dir. Le travail collaboratif : une innovation générique. Paris : Harmattan, 2009.

Ministère de l'Education nationale et de la formation professionnelle. Les orientations pédagogiques de l'enseignement du français dans le cycle secondaire collégial. Maroc, 2009.

Plane, Sylvie. « Stratégies de réécriture et gestion des contraintes d'écriture par des élèves de l'école élémentaire : ce que nous apprennent des écrits d'enfants sur l'écriture ». Rivista Italiana de Psicolinguistica Applicata anno 3 : 1 (2003) : 57-77.

Reuter, Yves. Enseigner et apprendre à écrire. Construire une didactique de l'écriture. Paris : ESF, 1996.

\footnotetext{
NOTES

${ }^{1}$ Nous nous intéressons au cycle collégial, parce que les stagiaires avec lesquels nous avons réalisé cette expérience étaient destinés à enseigner dans ce cycle.

${ }^{2}$ Selon J. Giasson, «le lecteur exprime des réflexions qui démontrent qu'il est engagé dans un processus de recherche de compréhension et d'autoévaluation ». Ce sont là les stratégies métacognitives ou encore les processus métacognitifs.

${ }^{3}$ La pédagogie du projet abolit l'émulation et favorise l'esprit du groupe moyennant la coopération, d'où l'intérêt d'attribuer des pseudonymes qui devraient mettre en avant «l'apprenant-scripteur » et occulter ainsi «l'apprenant-personne ». Chose qui participe à esquiver les problèmes de timidité et encourage les élèves en difficulté à participer sans avoir peur d'être jugés ou raillés par leurs camarades.
} 